\title{
Anisotropic nanostructured magnets by magnetic-field-assisted processing
}

\author{
Chuan-bing Rong, Van Vuong Nguyen, and J. Ping Liu ${ }^{\text {a) }}$ \\ Department of Physics, University of Texas at Arlington, Arlington, Texas 76019, USA
}

(Presented 19 January 2010; received 29 October 2009; accepted 1 December 2009; published online 15 April 2010)

\begin{abstract}
It has been a great challenge to produce anisotropic nanocrystalline bulk magnets. In this paper, we report our recent work in preparing anisotropic bonded magnets consisting of $\mathrm{SmCo}_{5}$ nanocrystalline particles. The work involved synthesis of nanocrystalline hard magnetic $\mathrm{SmCo}_{5}$ particles by ball milling in the presence of a magnetic field. Structural and magnetic characterizations show the alignment of the c-axis of the $\mathrm{SmCo}_{5}$ grains upon the magnetic-field-assisted ball milling, while the grain size was controlled under $15 \mathrm{~nm}$. The formation of the texture may be attributed to the effect of magnetic fields on the grain orientation during the cold welding process during ball milling. The as-milled particles were then compacted under a magnetic field with a binder to form bonded bulk $\mathrm{SmCo}_{5}$ nanostructured magnets. X-ray diffraction patterns show c-axis alignment in the bulk magnets, which was consistent with the magnetic measurements. () 2010 American Institute of Physics. [doi:10.1063/1.3337656]
\end{abstract}

\section{INTRODUCTION}

Nanostructured permanent magnetic materials have drawn considerable attention since they could potentially provide giant energy products. ${ }^{1,2}$ However, this potential can be realized only if the nanoscaled grains of the hard magnetic phases can be aligned to form anisotropic magnets. Extensive experimental effort has been devoted to achieve a high performance nanocomposite magnet in the past two decades. Unfortunately, the nanocomposite materials prepared by traditional methods, such as melt spinning and mechanical alloying, are always isotropic, ${ }^{3-9}$ which gives lower energy products as compared to the values predicted theoretically. For bulk nanocrystalline magnets, it turns out to be even more challenging than for the ribbons or powders to produce the textured microstructure with oriented nanograins of the hard magnetic phases. The traditional techniques to produce anisotropic bulk magnets, such as sintering and hot deformation, are associated with long thermal exposure and thus are not suitable to prepare anisotropic bulk magnets with nanostructured morphology due to excessive grain growth. Recently, several studies were reported on an approach to fabricate anisotropic micron- or submicron-scale particles containing aligned nanoscaled grains by magnetic-field and surfactant-assisted ball milling. ${ }^{10,11}$ The surfactants help reduce the particle size, while the magnetic field improves the grain alignment within each particle. This kind of anisotropic particles can be used as building blocks for anisotropic bulk magnets. In this work, we report on the effect of magnetic field on the formation of anisotropic hard magnetic particles with nanoscale grains and the preparation of bulk nanostructured anisotropic bonded $\mathrm{SmCo}_{5}$ magnets by compacting the particles in the presence of a magnetic field.

${ }^{a)}$ Electronic mail: pliu@uta.edu.

\section{EXPERIMENTAL DETAILS}

The commercial $\mathrm{SmCo}_{5}$ raw powders of about $45 \mu \mathrm{m}$ were first mixed with organic liquid in the glovebox. The liquid medium was used to reduce the particle size. The mixture was ground in a milling vial with balls in a ball-milling machine. The vial and balls are made of nonmagnetic stainless steel. The weight ratio of powders to balls is about 1:10. Two diametrical oriented arc-segment permanent magnets were fixed around the vial to create a quasihomogeneous magnetic field of about $2.5 \mathrm{kG}$. For comparison, the raw powders were also milled in the absence of a magnetic field, keeping other conditions constant. For characterizing the anisotropy, the milled particles were mixed with appropriate epoxy and then aligned under a magnetic field of about 20 kG. To study the effect of milling medium on the alignment degree of the particles, we choose three different types of liquids to be mixed with $5 \mathrm{~g} \mathrm{SmCo}$ powders: (1) $5 \mathrm{ml}$ heptane (99.8\% purity), (2) $5 \mathrm{ml}$ heptane and surfactants $(0.6 \mathrm{ml}$ oleic acid, $90 \%$ purity, and $0.6 \mathrm{ml}$ oleyl amine, $98 \%$ purity), and (3) $0.25 \mathrm{~g}$ phenylene sulfide (PPS) with $7 \mathrm{ml}$ xylene. The milled particles using PPS were also compacted under a magnetic field of about $18 \mathrm{kG}$ to produce a bulk bonded SmCo nanostructured magnets where PPS serves as a binder. The crystalline structure was characterized by a Rigaku Ultima IV x-ray diffraction (XRD) using $\mathrm{Cu} K \alpha$ radiation. Magnetic properties were measured with a superconducting quantum interference device magnetometer with a maximum applied field of $70 \mathrm{kOe}$.

\section{RESULTS AND DISCUSSION}

Figure 1(a) compares the XRD patterns of the aligned particles after surfactant-assisted ball milling for $4 \mathrm{~h}$ with and without a magnetic field. It was observed that the intensity of the (001) and (002) peaks of the particles milled in the presence of a magnetic field is higher than that in the absence of a magnetic field, while other peaks, such as (101), (110), and (111), have the same intensity. This indicates that the 

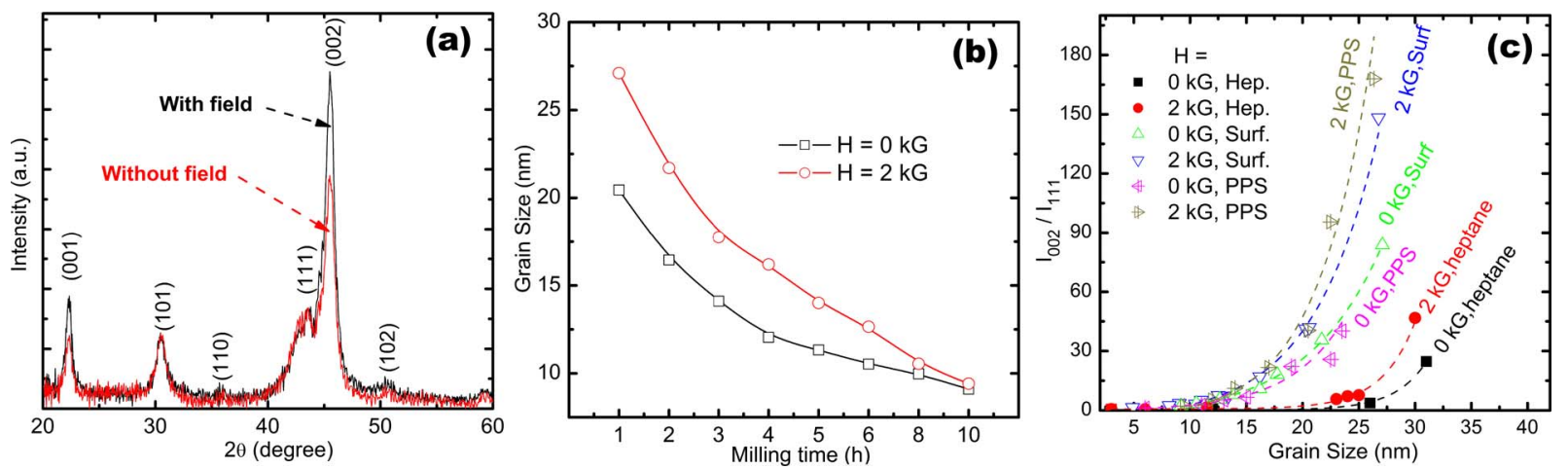

FIG. 1. (Color online) (a) XRD patterns of the aligned particles milled for $4 \mathrm{~h}$ with and without magnetic field. (b) Dependence of the grain size on the milling time of the particles milled with and without magnetic field. (c) Dependence of XRD peak intensity ratio of (002) to (111) on the grain size of the aligned particles after ball milling with different conditions.

particles milled in the presence of a magnetic field can be aligned more easily than particles milled in the absence of a magnetic field, which agrees with the previously reported results on magnetic-field milling of $\mathrm{NdFeB}$ and $\mathrm{SmCo}$ particles. ${ }^{10,11}$ However, it should be noted that magnetic fields may change the ball-milling efficiency. As shown in Fig. 1(b), the grain size of the particles milled under the magnetic field is bigger than that of regular milling, which means that the milling efficiency is lowered upon applying a magnetic field possibly due to the agglomeration of the particles under the influence of a magnetic field. It is therefore more reasonable to compare the degree of alignment of the particles having similar grain size.

Figure 1(c) shows the dependence of the intensity ratio of (002) to (111) peaks on the grain size of the aligned particles, which is an indicator of the magnetocrystalline alignment. Here, three series of samples are compared with particles milled with different liquid media. It was found that the degree of alignment of the particles milled in the presence of a magnetic field was always higher than that of the particles milled in the absence of a magnetic field for all the three milling medium. The mechanism of the texture formation may be attributed to the effect of magnetic fields on the grain orientation during the cold welding process in the ballmilling process. ${ }^{10}$ It was also interesting to observe that the grain size of the milled particles with the assistance of surfactant and binder is significantly smaller than that of the particles milled with only heptane, which should be attributed to the fact that surfactant or binder helps reduce the particle size.

To verify the effect of magnetic field on the alignment of grains of the milled particles, the demagnetization curves of the aligned particles were measured along the directions parallel and perpendicular to the c-axis. An appreciable difference was observed between the two demagnetization curves, as shown in Fig. 2(a), indicating that the particles are aligned. To quantitatively characterize the degree of grain alignment, one can compare the remanence along the aligned direction, $\mathrm{M}_{\mathrm{r}}(\|)$. Theoretically, the remanence ratio, $\mathrm{m}_{\mathrm{r}}(\|)$ $=\mathrm{M}_{\mathrm{r}}(\|) / \mathrm{M}_{\mathrm{s}}$, is equal to $\frac{1}{2}$ for assemblies of randomly oriented particles without interactions, i.e., Stoner-Wohlfarth particles, while it is equal to 1 for a perfectly aligned particle assembly. Practically, $\mathrm{m}_{\mathrm{r}}(\|)$ of the aligned particles is in between $\frac{1}{2}$ and 1 . A larger $m_{r}(\|)$ means a higher degree of grain alignment. Figure 2(b) shows the dependence of $\mathrm{m}_{\mathrm{r}}(\|)$ on the grain size of the aligned particles under different ball-milling conditions. It was found that $\mathrm{m}_{\mathrm{r}}(\|)$ of the particles milled in the presence of a magnetic field is higher than that of regular particles milled in three different media, which confirms the magnetic-field effects during ball milling as analyzed by XRD patterns. It also agrees with the XRD analysis that the particles milled with surfactants/PPS have a better grain alignment than those milled with only heptane if one com-
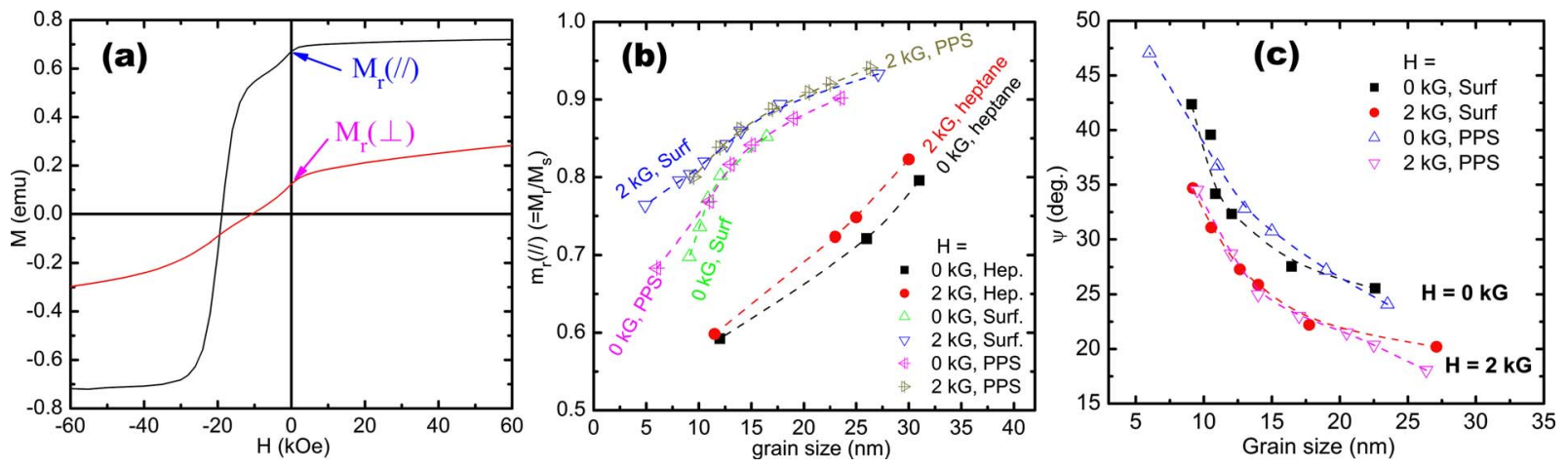

FIG. 2. (Color online) (a) Demagnetization curves of the aligned particles milled for $1 \mathrm{~h}$, which are measured along the directions parallel and perpendicular to the c-axis. (b) Dependence of the remanence ratio $\mathrm{m}_{\mathrm{r}}(\|)$ on the grain size of the particles milled with and without magnetic field. (c) Dependence of the average misalignment angle on the grain size of the particles milled with and without magnetic field. 

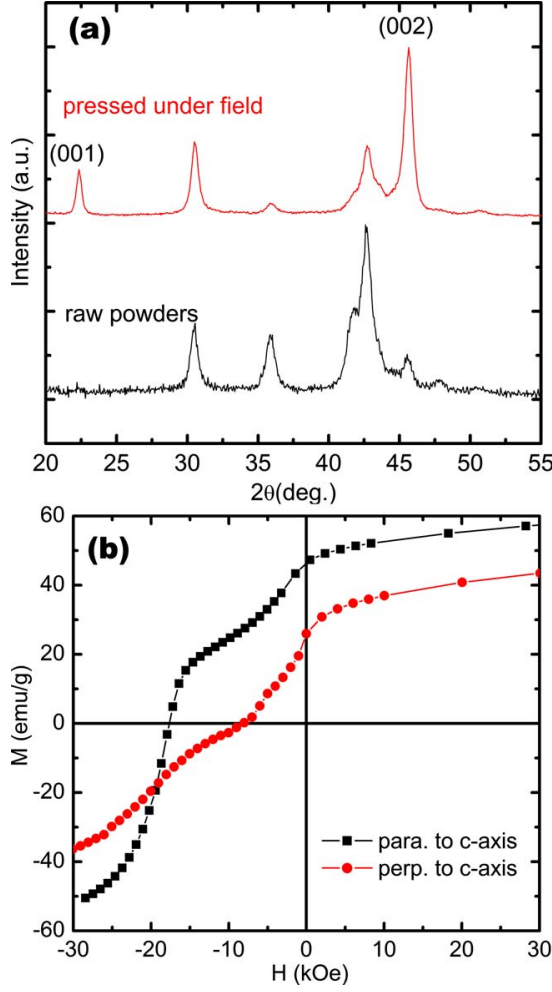

FIG. 3. (Color online) (a) XRD pattern of the compacted bulk magnet prepared under magnetic field from the anisotropic particles, which was compared with that of isotropic milled particles. (b) Demagnetization curves of the bulk magnets with grain size of about $15 \mathrm{~nm}$ measured parallel and perpendicular with respect to the c-axis.

pares the particles with same grain size. The alignment degree of the particles can be more accurately described by the average misalignment angle, $\psi=\arctan \left[2 \mathrm{M}_{\mathrm{r}}(\perp) / \mathrm{M}_{\mathrm{r}}(\|)\right]$, where $\mathrm{M}_{\mathrm{r}}(\perp)$ is the remanence perpendicular to the direction of the easy axis, which was proposed by Fernengel et $a l .{ }^{12}$ Figure 2(c) shows the dependence of the misalignment angle on the grain size of the particles milled with surfactants and PPS. It was found that $\psi$ of the particles milled in the presence of a magnetic field is smaller than that of the regularly milled particles, which again confirms that the field-milled particles have a higher degree of alignment. It should be noted that the degree of grain alignment always decreases with decreasing grain size due to the fact that long ball milling is inclined to randomly orient the grains, and incoherence in grain boundaries reduces the alignment.

Based on the anisotropic particles with nanoscale grains, it is possible to produce a bulk nanostructured magnet by compaction of the particles in the presence of a magnetic field. The magnetic field aligns the magnetic particles during which the compaction consolidates the aligned particles. Figure 3(a) shows the XRD patterns of the bulk magnets prepared by magnetic-field compaction of the particles milled with 4.8 wt \% binder in a field of about $18 \mathrm{kG}$ and a pressure of about $400 \mathrm{MPa}$. The intensity of the (002) peak of the XRD patterns was significantly enhanced and the intensity ratio of (002) to (111) peak is about 2.4, compared to 0.25 of typical patterns of isotropic magnets. In addition, the grain size estimated by the Scherrer formulas is only about $15 \mathrm{~nm}$ without considering the strain effect but just instrumental correction. Figure 3(b) shows the demagnetization curves of the bulk magnet samples cut (geometry has negligible demagnetization factor) and measured parallel and perpendicular to the easy axis. The difference between the curves confirms the formation of texture in the bulk nanostructured $\mathrm{SmCo}_{5}$ magnets. It should be mentioned that the XRD patterns and demagnetization curves are representative for at least five experiments. The density of the bulk magnet is about $6.0 \mathrm{~g} / \mathrm{cm}^{3}$ and the energy product is about 2.6 MG Oe. Further improvement of magnetic properties can be achieved by reducing the amount of binder and by increasing the compact density of the bulks.

\section{CONCLUSIONS}

In conclusion, bulk anisotropic nanostructured magnets were prepared by compacting in the presence of a magnetic field of anisotropic nanostructured $\mathrm{SmCo}_{5}$ particles prepared by the magnetic-field-assisted ball milling with the assistance of surfactants or binder. The external magnetic field facilitates the formation of the anisotropic particles and bulk magnets, as conformed by XRD and magnetic property analysis. In addition, it was found that surfactant and binder reduce grain size while keep a high degree of grain alignment in the milled particles, compared with the milled particles with only heptane. This processing technique is promising for industrial scale production of anisotropic nanostructured bulk magnets in the future.

\section{ACKNOWLEDGMENTS}

This work was supported by U.S. DoD/MURI under Grant No. N00014-05-1-0497 and by the Center of Nanostructured Materials and Characterization Center for Materials and Biology at the University of Texas at Arlington.

${ }^{1}$ R. Skomski and J. M. D. Coey, Phys. Rev. B 48, 15812 (1993).

${ }^{2}$ C. B. Rong, H. W. Zhang, R. J. Chen, S. L. He, and B. G. Shen, J. Magn. Magn. Mater. 302, 126 (2006).

${ }^{3}$ R. Coehoorn, D. B. de Mooij, and C. de Waard, J. Magn. Magn. Mater. 80, 101 (1989).

${ }^{4}$ A. Manaf, R. A. Buckley, and H. A. Davis, J. Magn. Magn. Mater. 128, 302 (1993).

${ }^{5}$ H. A. Davies, J. Magn. Magn. Mater. 157-158, 11 (1996).

${ }^{6}$ J. Bauer, M. Seeger, A. Zern, and H. Kronmuller, J. Appl. Phys. 80, 1667 (1996).

${ }^{7}$ D. Goll, M. Seeger, and H. Kronmuller, J. Magn. Magn. Mater. 185, 49 (1998).

${ }^{8}$ J. Zhang, S. Y. Zhang, H. W. Zhang, and B. G. Shen, J. Appl. Phys. 89, 5601 (2001).

${ }^{9}$ C. B. Rong, H. W. Zhang, J. Zhang, X. B. Du, S. Y. Zhang, and B. G. Shen, J. Appl. Phys. 97, 033907 (2005).

${ }^{10}$ N. Poudyal, B. Altuncevahir, V. Chakka, K. Chen, T. D. Black, J. P. Liu, Y. Ding, and Z. L. Wang, J. Phys. D: Appl. Phys. 37, L45 (2004).

${ }^{11}$ P. Saravanan, R. Gopalan, N. V. R. Rao, M. M. Raja, and V. Chandrasekaran, J. Phys. D: Appl. Phys. 40, 5021 (2007).

${ }^{12}$ W. Fernengel, A. Lehnert, M. Katter, W. Rodewald, and B. Wall, J. Magn. Magn. Mater. 157-158, 19 (1996). 